\title{
A Human in the Loop Corrective Maintenance Methodology Using Cross Domain Engineering Data of Mechatronic Systems
}

\author{
Milan Vathoopan, Benjamin Brandenbourger, Alois Zoitl \\ fortiss $\mathrm{GmbH}$ \\ Guerickestr. 25, 80805 Munich, Germany \\ \{vathoopan, brandenbourger, zoitl\}@fortiss.org
}

\begin{abstract}
Technology trends and market changes force modern manufacturing companies to employ complex, standalone mechatronic components for automating their production. The complexity of mechatronic components induces adverse effects on their corrective maintenance. Handling downtime of these components requires knowledge of system composition, effect of external and internal disturbances on the component etc. Recognizing the human efficiency in industrial maintenance, this paper introduces a human centered corrective maintenance methodology for mechatronic components leveraging the cross disciplinary engineering data of these components. A 3D simulation model of the components' cyber-physical principles derivable from its engineering data is proposed for supporting the human doing maintenance action. A software intensive system architecture and application methods substantiating the concept are advocated.
\end{abstract}

\section{INTRODUCTION}

Modern manufacturing companies manage the dynamic customer requirements and market challenges with automation facilities equipped with mechatronic components (systems) [1]. Different disciplines are involved in the development, upgrades or adaptation of mechatronic components. Model based and standardized engineering approaches are being established for managing the complexity and improving inter disciplinary interaction in the development of mechatronic systems. [2]. Once commissioned, many external and internal disturbances like pressure, temperature, friction etc. can affect the normal operation of mechatronic components and can lead to a downtime [3]. The corrective maintenance of a mechatronic component require specific disciplinary knowledge like electromechanical and control principles, interdisciplinary interactions and interdependence within them [4]. Rapid and correct decision making, based on the system composition [5], its engineering and dependence of the system on external and internal factors is also required for taking them back to production. Because of this complex nature preventive or prognostic maintenance techniques are mainly investigated in case of mechatronic components, in order to avoid any downtime [6]. Corrective maintenance practices or methodologies still remain as an open challenge.

Identifying the human ability in revamping the industrial maintenance, this paper introduces a human in the loop correc-

978-1-5090-1314-2/16/\$31.00 (c) 2016 IEEE tive maintenance methodology applicable to mechatronic components. The proposed methodology aims to aid the human doing the corrective action, considering the complex systemic and engineering characteristics of mechatronic components. A 3D simulation of the components' cyber-physical view [7], detailing the basic working principles is put forwarded for analyzing the system. Coordination of different disciplines, embedded with external and internal dependencies is also provided for rapid decision making in case of a downtime. The mechatronic data model of the component applied for its model based development is leveraged here for producing this visual interaction environment. The data model of a component is capable of detecting runtime errors or inconsistencies in the field. The proposed corrective maintenance methodology is applied at the time when an error or inconsistency leading to a downtime is detected. A software intensive system architecture and application methods are presented based on the concept. Detailed engineering aspects of a component based on its mechatronic data model is out of scope of this paper.

The rest of this paper is organized as follows. Section II gives an overview of the required background knowledge leading to the proposed concept. In Section III, the functional requirements are deduced and the methodology is presented. Section IV details the simulation model and system architecture. Section V conclude the paper giving an overview of ongoing work and expected results.

This work has been supported by the German Federal Ministry of Economic Affairs and Energy (BMWi) as part of the research program "Autonomik Industrie 4.0" in the research project OPAK (Funding no.01MA13012F).

\section{BACKGROUND}

\section{A. Model Based Engineering of Mechatronic Components}

Mechatronic components generally comprises mechanical, electrical entities synchronized by complex software and contribute a functional requirement in an automation system [1]. A model based development of a mechatronic component thus require identifying the interdependence among different disciplines involved, coordinating them and maintaining consistency throughout its life cycle. Since the function of the component remains the same for all disciplines, a function oriented development is recommend in [8]. Behavior based 
approaches exist for a function oriented development of a component, where behavior represents the expected functioning of a component. Botaschanjan et al. [9] propose an integrated hybrid behavioral model for checking collisions and valid transformations of a component during development. They Interlink internal conditions and signals with volumes and operations for this purpose. Legat et al. [10] describe an interface behavior modeling, where static interfaces are connected with a hybrid semantic behavior model considering requirements, system and process viewpoints. They apply the concept for verifying the functional conformance of a model at different stages of development. Building upon the above works a behavior model is used in our approach for interlinking different disciplines in a mechatronic data model.

As a first step, extending our previous work [11] a skeleton of a mechatronic component model is implemented in the standard automation data exchange format AutomationML (AML) [12]. The AML model built for facilitating tool integration and inter disciplinary integration is shown in Fig. 1. Here functionality of a device is expressed in terms of skills [13] offered by the component and behavior model implemented in PLCopen XML. The component hierarchy is implemented with internal elements and internal links. Multidisciplinary information are embedded with the help of external and internal interfaces.
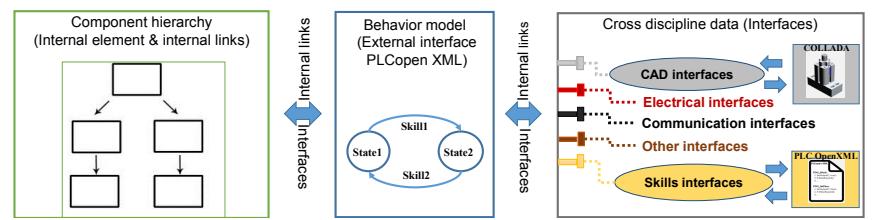

Fig. 1. Skeleton of a mechatronic component implemented in AutomationML

\section{B. State of the Art Maintenance and Management Practices}

Lee et al. [6] describes that intelligent, self adaptable and resilient machines are envisioned in the future. Lee et al. also points out that the most strategically evolved term in maintenance in recent times is prognostic and health management technologies. Based on these technologies nowadays predictive and preventive methods of maintenance prevail [5]. Most of the researches in this directions are towards making the system self aware using machine learning algorithms and self dissipative based on decision algorithms. Chen et al. [14] propose a virtual information infrastructure for the development of evolvable production systems which is also applicable for anomaly detection in the engineering process. Situation of corrective maintenance of a system is not well explored so far.

The human role in improving the effectiveness and efficiency of maintenance by adding human specific knowledge and skills is explained in [15]. And their results shows that these human specific skills cannot be obtained solely through data analysis of sensors. The potential of visual computing for industrial device management is explained in [16]. Above works shows the importance of having a human involvement and facilitating a visual computing environment as a medium for human machine interaction in industrial environment.

\section{Corrective maintenance of A Mechatronic COMPONENT USING ITS ENGINEERING DATA}

\section{A. Deduction of Requirements for Corrective Maintenance of a Mechatronic Component}

In order to deduce an efficient and effective maintenance process a number of context, human and function related requirements have to be considered. As discussed in the above section human involvement (R1) is necessary to handle complexity of mechatronic components and take rapid and correct decisions [15]. A visual computing environment (R2) is recommended [16] as a better human machine interaction medium. To represent the interdependence of the involved disciplines to the human, a behavior based interlinking (R3) as used in model based development needs to be provided [10], [9]. Basic working principles and its dependencies with external and internal disturbances [3], [4] have to be made available to the user (R4). The maintenance approaches are nowadays prevailed by preventive and prognostic techniques [5], which can give information like when, where, and why a failure occur. These information must be available to the user if necessary (R5). On the other hand a corrective maintenance action can provide inputs to the preventive and diagnostic data bases (R6). Different methods of debugging have to be provided based on the skills of the personnel such as providing a recipe or solve using their own analytical and decision making skills (R7).

\section{B. Leveraging the Engineering Data Model of a Mechatronic Component for its Corrective Maintenance}

A basic AML model of a mechatronic component in the application context is shown in Fig. 2 (a). Behavior of the component (expected functionality of the component) acts as the core of the model and interlinks the control code (software domain) and 3D kinematics (mechanical domain). The interaction between domain-specific models (software and mechanical here) have to traverse the core behavioral model in a component model. Each discipline works in parallel on its domain-specific tasks and creates interdependence to the central behavioral model. This interdependence and interlinking is used in such a way that the application programming interface (API) is encapsulated with skills and behavior it holds.

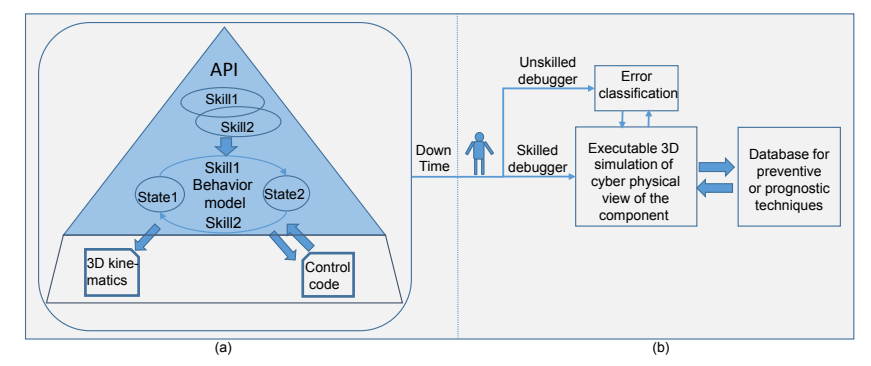

Fig. 2. Proposed methodology for corrective maintenance based on the engineering approach: (a) Engineering data model of a mechatronic component in application context, (b) Human in the loop corrective maintenance scenario. 


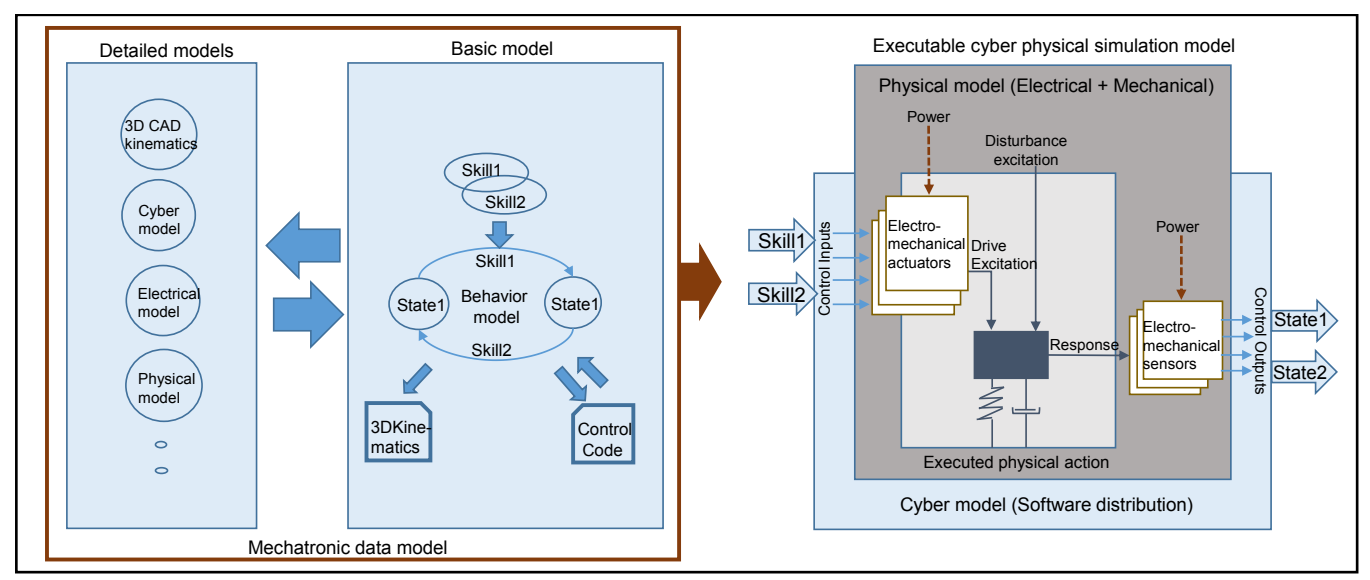

Fig. 3. Deriving an executable 3D cyber-physical simulation model of a mechatronic component based on [17]

A detailed and concrete model with domain specific details and real-time aspects is applicable during runtime of a component in the field. During runtime the core behavior model is influenced by domain-specific models in such a way, that any inconsistency or error can be detected (e.g., a sensor does not change its state as expected, an error has occurred in the mechatronic system). This is done by comparing the expected behavior of a component with the real behavior influenced by sensor values. The proposed corrective maintenance methodology is applicable at the time when an error leading to a downtime is detected. Based on the requirements listed above, a maintenance methodology is envisioned based on the runtime mechatronic data model of the component as shown in Fig. 2 (b).

When a downtime occurs, a human responsible for the maintenance inspect the component. The person can be skilled or unskilled to handle a downtime. The application methodology allows the skilled debugger to use a visual interface to the component and solve the issue using his analytical and debugging skills. The visual interface provides adequate system details and engineering data helping the human for debugging in this case. On the other hand an error classification and debugging recipes are created for known errors for helping an unskilled person. The recipe in this case is again connected to the visual interface and provides a real time system view that help the unskilled person in learning the system and the debugging recipe. The visual interface is linked with the database created for prognostic techniques, which allows accessing any data from the database or vice versa.

\section{Proposed simulation Model and System ARCHITECTURE}

A. Deriving an Executable $3 D$ Simulation Model from the Engineering Data

Sec. III-B detailed the proposed method of application. Mapping further requirements on to the medium of interaction, a cyber-physical simulation model of the component encapsulated with its behavior and skills is envisaged. The executable 3D simulation incorporates the basic working principles and inter disciplinary interaction within the component.
As shown in Fig. 3, initially the basic model of a component is considered with its behavior as the core. At this stage an exemplary behavior consists of two states and two skills. From State1 it makes a transition to State2 when Skill1 is executed. And from State2 it makes a transition back to State1 if Skill2 is executed. The runtime model of a component contains detailed domain specific models like physical model, kinematic model, electrical model, etc. and temporal information. The interdependence and interlinking is represented within the behavior of the component.

For demonstrating the concept, an exemplary cyber-physical simulation model based on [17], derivable from the mechatronic data model of component is shown in Fig. 3. It contains the executed physical action producing the required functionality of the component, control inputs and outputs on top. Further it encompasses electromechanical actuators to actuate the physical action and sensors to monitor the action. Disturbances or excitations are also represented when sufficient information is available. The cyber model encapsulating the physical model synchronize the interdisciplinary interaction and engineering of the component with the behavior model characterizing the component. These inter disciplinary aspects are again connected with the basic behavior model and other required engineering data. The derived executable 3D simulation model functions same as the real component, with two states State1 and State2 and two skills Skill1 and Skill2.

\section{B. System Architecture and Working Principle}

The system architecture presented in Fig. 4 is adhering to the application methodology described in the previous sections. The real component is represented as a black box assuming that the internal working is not explicitly visible to the external user. The components are accessible to the external world by the skills offered (Skill1, Skill2) and monitored by the traversed states (State1, State2). The 3D simulation of the component represents a mirror image of the real component, but it gives actual cyber-physical internal view of a component and other details as mentioned above. The 3D simulation runs in real-time in a cloud or back end parallel to the real component. When the behavior model of the real component 
makes a state transition the same is reflected in the simulated component. Thus an easily perceivable and detailed model of the component encapsulated with its functionality is available to the user. The simulated component is able to connect with the data maintained for preventive and prognostic techniques and error classification. The person who is responsible for the maintenance is able to get the simulated view of the component with consumer devices like smart phone, tablets, glass etc. The interacting device can be applied from the field directly or from remote locations that give the simulated component.

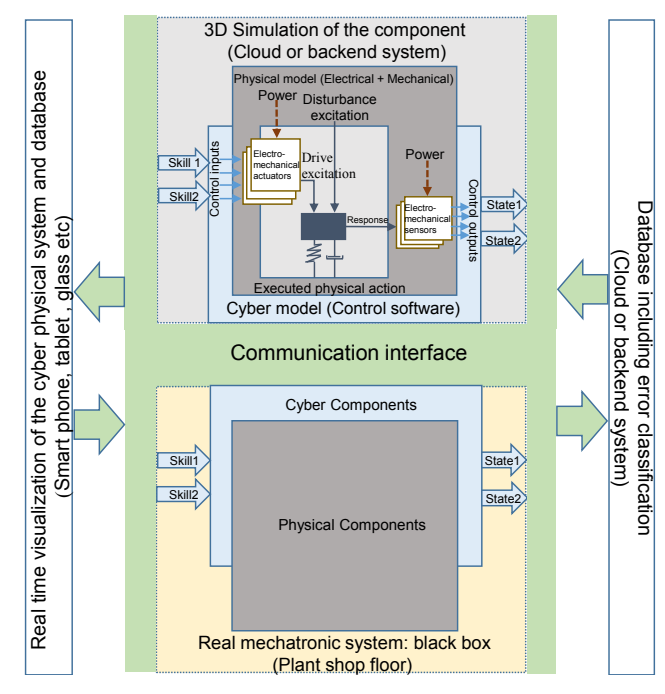

Fig. 4. Proposed system architecture

\section{CONClusion And Future WORKS}

This paper proposes a methodology addressing one of the key challenges in industrial automation domain, corrective maintenance of mechatronic components. The concept introduced here put forward the following advantages. It leverages the engineering data aggregated during its development phase, which is normally employed only till initial commissioning or for reconfiguration. The proposed concept takes the human in the loop and creates an efficient and effective maintenance process. An interactive visual environment aiding the human to solve issues related to the component is propounded. Instead of merely depending on machine learning and decision support algorithms, it gives challenge to human and expected to give work satisfaction. And the concept enhances the preventive and prognostic techniques, which are widely researched on these days by giving corrective action inputs.

As a first step of the implementation, a basic description of mechatronic components with behavior model as the core is prepared in AML. A detailed AML model of a component applicable during runtime is under investigation. Modelica is being evaluated for implementing cyber-physical simulations of mechatronic components. The following results are expected in the future. The AML data can be directly converted to an OPC UA name space [18] giving standard interfaces to a component. Different models of a component such as physical model, kinematic model etc. will be interlinked in AML with behavior model as the core. The same AML data model can be employed to prepare the simulation model using Modelica. The technology enhancements in the visual engineering such as augmented reality can be used to enrich the visual human machine interface. Clearing up the above described challenges, scalability of the concept to a system composed of mechatronic components and an industrial use case analysis are envisioned.

\section{REFERENCES}

[1] P. Stich and G. Reinhart, "Mechatronic Sketching of Manufacturing Systems Using Physically Based Models: A Novel Approach for Simulation Based Systems Engineering," in IEEE Symp. on Industrial Electronics \& Applications(IndIn), 2013.

[2] B. Vogel-Heuser, C. Legat, J. Folmer, and S. Rösch, "Challenges of Parallel Evolution in Production Automation Focusing on Requirements Specification and Fault Handling," at-Automatisierungstechnik, vol. 62, no. 11, 2014.

[3] W. M. Goble, Control Systems Safety Evaluation and Reliability. ISA, 2010.

[4] R. Isermann, "Mechatronic SystemsInnovative Products With Embedded Control," Control Engineering Practice, vol. 16, no. 1, 2008.

[5] J. Lee and B. Bagheri, "Cyber-Physical Systems in Future Maintenance," in 9th WCEAM Research Papers. Springer, 2015.

[6] J. Lee, B. Bagheri, and H.-A. Kao, "A Cyber Physical Systems Architecture for Industry 4.0 Based Manufacturing Systems," Manufacturing Letters, vol. 3, 2015.

[7] R. R. Rajkumar, I. Lee, L. Sha, and J. Stankovic, "Cyber Physical Systems: The Next Computing Revolution," in Proceedings of the 47th Design Automation Conf. ACM, 2010.

[8] P. Ovtcharova, I. Jivka, M. Marinov, D. Gutu, D. Szots, A. Simonyi et al., "Representation of Cross-Domain Design Knowledge Through Ontology Based Functional Models," in Proceedings of the 18th International Conf. on Engineering Design (ICED 11), Lyngby/Copenhagen, Denmark, 2011.

[9] J. Botaschanjan, B. Hummel, T. Hensel, and A. Lindworsky, "Integrated Behavior Models for Factory Automation Systems," in IEEE Conf. on Emerging Technologies \& Factory Automation(ETFA). IEEE, 2009.

[10] C. Legat, J. Mund, A. Campetelli, G. Hackenberg, J. Folmer, D. Schütz, M. Broy, and B. Vogel-Heuser, "Interface Behavior Modeling for Automatic Verification of Industrial Automation Systems' Functional Conformance," at-Automatisierungstechnik, vol. 62, no. 11, 2014.

[11] B. Brandenbourger, M. Vathoopan, and A. Zoitl, "Engineering of Automation Systems Using a Metamodel Implemented in AutomationML," in IEEE Conf. on Industrial Informatics (IndIn). IEEE, 2016.

[12] R. Drath, A. Lüder, J. Peschke, and L. Hundt, "AutomationML-The Glue for Seamless Automation Engineering," in IEEE International Conf. on Emerging Technologies \& Factory Automation(ETFA). IEEE, 2008.

[13] M. Bengel, "Modelling Objects for Skill Based Reconfigurable Machines," in Virtual International Conference on Innovative Production Machines and Systems IPROMS, 2007.

[14] D. Chen, A. Maffei, J. Ferreirar, H. Akillioglu, M. R. Khabazzi, and $\mathrm{X}$. Zhang, "A virtual environment for the management and development of cyber-physical manufacturing systems," IFAC-PapersOnLine, vol. 48, no. 7, 2015.

[15] N. Duarte Filho, S. Botelho, M. Bichet, R. P. dos Santos, G. Schroeder, R. Nagel, D. Espíndola, and C. E. Pereira, "Human Computer Interface (HCI) for Intelligent Maintenance Systems (IMS): The Role of Human and Context," in Engineering Asset Management-Systems, Professional Practices and Certification. Springer, 2015.

[16] J. Posada, C. Toro, I. Barandiaran, D. Oyarzun, D. Stricker, R. De Amicis, E. B. Pinto, P. Eisert, J. Dollner, and I. Vallarino, "Visual Computing As a Key Enabling Technology for Industrie 4.0 and Industrial Internet," Computer Graphics and Applications, IEEE, vol. 35, no. 2, 2015.

[17] C. W. De Silva, Sensors and Actuators: Engineering System Instrumentation. CRC Press, 2015.

[18] R. Henßen and M. Schleipen, "Interoperability Between OPC UA and AutomationML," Procedia CIRP, vol. 25, 2014. 